\title{
The effects of alternating tactile and acoustic stimuli on habituation of the human eyeblink reflex
}

\author{
WILLIAM A. DYCUS and ALICE S. POWERS \\ St. John's University, Jamaica, New York
}

\begin{abstract}
In the present investigation, we explored the effects of altemating sensory stimuli of two modalities on habituation to those stimuli. The subjects in two control groups participated in 25 trials in which they were exposed to either cutaneous (stimulation of supraorbital nerve) or acoustic (white noise burst) stimuli with a 5-sec interstimulus interval (ISI). The subjects in the experimental group participated in 50 trials in which they were exposed to alternating cutaneous and acoustic stimuli at 2.5-sec ISIs. Habituation in the experimental group, in which the cutaneous stimulus was alternated with acoustic stimuli, was expected to be more rapid than habituation in the control groups, in which the cutaneous stimulus was presented alone, due to the indirect stimulation of the supraorbital nerve by the eyeblinks was elicited by each acoustic stimulus. As predicted, alternating cutaneous and acoustic stimuli enhanced habituation to the cutaneous stimulus. Habituation to the acoustic stimulus was not increased by the interpolation of cutaneous stimuli. These results support the hypothesis that blinking to an acoustic stimulus will generate cutaneous stimuli that interact with direct cutaneous stimulation to engage modulatory processes.
\end{abstract}

Habituation is the reduction of a reflex reponse to a stimulus as a result of repeated presentations of that stimulus. Just as the motor reflex is considered to be the simplest of stimulus- response mechanisms, habituation of a reflex response is considered by most researchers to be one of the simplest forms of learning (File \& Russell, 1972). Habituation has been demonstrated in a wide range of multicellular organisms, from the sea slug Aplysia (Kandel, 1978) to Homo sapiens (Sanes \& Ison, 1983). As a result, the study of habituation gives researchers a basis for understanding how humans acquire information and learn about their environment.

An eyeblink can be evoked by a number of different stimuli, such as pure acoustic tones (Tackmann, Ettlin, \& Barth, 1982), bursts of white noise (Graham, 1975), puffs of air to the skin around the eye (Evinger, Shaw, Peck, Manning, \& Baker, 1984; Reiter \& Ison, 1977), flashing lights (Tackmann et al., 1982), and electrical stimulation of the supraorbital branch of the trigeminal nerve (Powers, Schicatano, Basso, \& Evinger, 1997; Rimpel, Geyer, \& Hopf, 1982; Sanes \& Ison, 1983). In the present study, we investigated how blinks elicited by different forms of stimulation interact--specifically, how they influence each other during the process of habituation. We studied the effect of alternating acoustic and cutaneous stimulation on habituation, by comparing the rate of habituation of a group of subjects given both stim-

Address correspondence to A. S. Powers, Department of Psychology, St. John's University, 8000 Utopia Parkway, Jamaica, NY 11439 (e-mail: powersa@stjohns.edu). uli with those given only acoustic stimuli or only cutaneous stimuli.

A number of theories have been proposed to account for habituation (Groves \& Thompson, 1970; Sokolov, 1960; Wagner, 1979), but none has dealt specifically with the effect of alternating stimuli of two different modalities. Previous work in our laboratory (Dycus \& Powers, 1997) had shown, however, that the modalities of acoustic and cutaneous stimulation did not interact in a crosshabituation paradigm in which 30 cutaneous stimuli were given before the acoustic stimuli. Habituation to the acoustic stimuli followed the same course as for subjects who had not been previously exposed to the cutaneous stimuli. In the same experiment, we predicted that the stimuli would interact when the acoustic stimuli were given first. We made this prediction because the act of blinking to an acoustic stimulus activates the cutaneous afferents that elicit a blink (e.g., when air moves through the eyelashes or when the eyelid rubs against the cornea). Thus, habituation to cutaneous stimuli (as well as to acoustic stimuli) would have taken place during the presentation of acoustic stimuli, and when explicit cutaneous stimuli were subsequently presented, the sensory system would already have been partially habituated. This prediction was borne out: The subjects given acoustic stimuli first were already habituated to the cutaneous stimuli by the time of presentation.

The purpose of the present investigation was to extend these results and also to provide normative empirical data on habituation to cutaneous and acoustic stimuli. One experimental group and two control groups were tested. The control groups received repeated presentations of only 
one stimulus, either cutaneous or acoustic, with an intertrial interval (ITI) of $5 \mathrm{sec}$. The experimental group received alternating presentations of cutaneous and acoustic stimuli, with the interstimulus interval (ISI) exactly half that for the control groups $(2.5 \mathrm{sec})$. The eyeblink habituation rates for each modality in the experimental group were then compared with their respective control group's habituation rates to determine whether interjection of stimuli of another modality would affect habituation.

We predicted that habituation to the cutaneous stimuli would be more rapid in the alternating-stimuli group because those subjects would have been exposed to similar cutaneous stimuli when they had been presented with the acoustic stimuli. We expected little or no effect on habituation to the acoustic stimuli in the same group because the subjects would not have been exposed to acoustic stimuli when the cutaneous stimulus had been presented.

\section{METHOD}

\section{Subjects}

Thirty subjects were recruited through campus advertisement and from undergraduate and graduate courses. Of the 30 subjects in the experiment, 20 were female and 10 were male. The male subjects were evenly distributed among the three groups; the alternatingstimuli (ALT) and the acoustic-stimuli (AC-ONLY) groups had 3 males each, and the cutaneous-stimuli (SO-ONLY) group had 4. All subjects were adults (21-35 years), and none reported neurological problems.

Data from 6 additional subjects were discarded. Five of these subjects' data were not included because of equipment malfunction. The other subject was determined to be an extreme outlier, since the subject did not show any habituation to either the acoustic or the cutaneous stimuli.

\section{Apparatus}

The subjects were run in a quiet testing room $(1.5 \times 2.0 \mathrm{~m}$; background noise level, $50 \mathrm{~dB}$ ) that contained a comfortable chair and a small table with reading material. The testing equipment was located in an adjacent cubicle.

Stimulus presentation and data recording were performed automatically by an EMG data collection computer program. The acoustic stimuli were presented by Coulbourn Instruments equipment through Realistic binaural headphones (Nova 40). The cutaneous stimuli were presented by a WPI Stimulus Isolator (A360). The duration and rise-fall time of the acoustic stimuli were controlled by Coulbourn equipment, and the duration of the cutaneous stimuli was controlled by a WPI Pulsemaster timer (A300).

The EMGs were amplified by a differential ac amplifier (A-M Systems Model 1700 , band pass $-3 \mathrm{~dB}$ points at $300-5000 \mathrm{~Hz}$ ). They were collected, displayed, and stored online by a DataTranslation DT2801-A board and a personal computer. The EMGs were sampled at $4000 \mathrm{~Hz}$; the record length was set at $200 \mathrm{~ms}$, with the stimulus being triggered $10 \mathrm{~ms}$ after the beginning of each record. During the data storage process, the computer rectified the record, integrated the muscle activity, and stored each trial individually.

\section{Procedure}

The subjects were randomly assigned to one of three groups $(n=$ 10 ). The acoustic (AC) stimulus consisted of a $100-\mathrm{msec}$ whitenoise burst with near-instantaneous rise-fall time. The cutaneous stimulus consisted of electrical stimulation of the supraorbital (SO) branch of the trigeminal nerve (located just above the eye) and lasted $120 \mu \mathrm{sec}$. The AC-ONLY control group was presented with 25 tri- als of the $\mathrm{AC}$ stimulus with $5 \mathrm{sec}$ between trials. The SO-ONLY control group was exposed to 25 trials of stimulus presentation with $5 \mathrm{sec}$ between trials. The experimental (ALT) group was exposed to 50 trials with $2.5 \mathrm{sec}$ between trials, alternating back and forth between AC and SO stimuli. ALT subjects received 25 trials of the $\mathrm{AC}$ stimulus and 25 trials of the SO stimulus. Half of them were presented with an $\mathrm{AC}$ stimulus first (AC-SO); the other half was presented with an SO stimulus first (SO-AC). The subjects were not informed of their assigned experimental condition until they had completed the experimental procedure.

Each subject signed a written consent form that explicitly stated that the subject reserved the right to end his or her participation in the study at any time and was given a copy of the IRB guidelines for research using human subjects. The experimenter attached five Grass gold-plated 8-mm electrodes to different points around the right eye, using Grass electrode cream (EC-2) and paper first-aid tape. Two electrodes were used to record EMG output; one was affixed just inferior to the center of the right eye, and the other was affixed just lateral to the right eye. Two electrodes were used to present the SO stimulus; one was affixed just superior to the supraorbital notch of the right eye above the eyebrow, and the other was affixed approximately $1 \mathrm{~cm}$ above and slightly medial to the first one. The fifth electrode, attached at the center of the forehead, served as a ground.

After the subject was seated in the testing room, the experimenter connected the electrode wires and adjusted the headphones. The subject was then provided with reading material and was asked to read throughout the experiment. The investigator adjusted the level for both the $\mathrm{AC}$ and SO stimuli. In order to determine the stimulus threshold of the SO, the experimenter asked the subject to indicate each time he or she felt the stimulus. Beginning at $1.5 \mathrm{~mA}$, the experimenter presented an SO stimulus at intervals of $0.5 \mathrm{~mA}$ until the subject reported feeling the stimulus. The experimental level of the SO was then set at two times the stimulus threshold at which the subject had reported feeling the stimulus or at a minimum of $5.0 \mathrm{~mA}$ (duration was always $120 \mu \mathrm{sec}$ ). The subject was presented a single SO stimulus at the experimental level to test for pain or discomfort. None of the subjects reported either pain or discomfort at the experimental level; thus, no further adjustment was made. The $\mathrm{AC}$ stimulus was also tested to determine whether the volume was uncomfortable for the subject. Only 2 subjects reported the AC stimulus to be uncomfortable at the experimental level, so the volume was reduced slightly, until it was tolerable to those subjects (maximum $3-\mathrm{dB}$ reduction). Although the experimental levels of the SO varied widely between subjects $(5.0-10.0 \mathrm{~mA})$ because of differing thresholds, the AC levels remained relatively constant $(95 \mathrm{~dB})$. The same preexperimental procedures were followed for all subjects regardless of group assignment to maintain procedural consistency. Therefore, even the subjects who received only one stimulus type had the levels of both stimuli set prior to the experiment proper. In all cases, the stimulus presented first was tested first. Thus, for an SO-ONLY subject or an ALT subject whose experimental trials began with an SO stimulus, the experimenter tested and set the level of the SO first and then tested the level of the AC stimulus.

Once the experimental stimulus levels were set, the investigator informed the subject that the experiment proper was about to begin, that the subject did not need to respond verbally to any subsequent stimuli, and that the subject should just relax and read the magazines provided (e.g., National Geographic). The experiment proper lasted approximately $2.5 \mathrm{~min}$.

\section{EMG Analysis}

The EMG data were analyzed using an off-line computer analysis program developed by Craig Evinger at SUNY Stony Brook. The trials for each subject were analyzed individually. The program took the EMG data that were stored by the computer during the experiment and presented them trial by trial in graphic, rectified form. 
The program then converted the rectified amplitude of the blink response within a specified window of time into a single number for each trial. In this study, the window was set at $65 \mathrm{msec}$ and began approximately $15 \mathrm{msec}$ after the presentation of the stimulus, but this varied slightly among subjects because of variations in latency.

In order to control for differences in level of background noise in the EMG records, the baseline level of EMG was calculated for each subject and subtracted from the EMG amplitude for that subject. The background level was defined as the mean of baseline recordings over 10 trials. For each subject, 10 representative recordings were chosen over the course of the trial series. A $65-\mathrm{msec}$ window was used for the analysis and was placed near the end of the 200-msec recording. Only trials that showed no extra blink responses within the $65-\mathrm{msec}$ window were chosen. A mean of 10 trials was calculated for each subject; the mean was then subtracted from each trial in the series for that subject. If the result for any trial was a negative number, the amplitude was set to zero.

\section{Results}

Examples of a typical cutaneous and acoustic eyeblink response are presented in Figure 1. The electrically in- duced blink reflex is generally considered to consist of two components: a short-latency, short-duration ipsilateral burst of EMG activity (R1) and an EMG burst that is bilateral and longer in latency and duration ( R2; Kugelberg, 1952). Under the conditions of this experiment, only the R2 component was elicited reliably, as is shown in Figure la. The R2 component is more prominent and is observable even at relatively low intensities, whereas the $\mathrm{R} 1$ component occurs only at higher stimulus intensities. The acoustic blink reflex has only one observable component. Like the R2 component of the electrically induced blink reflex, it is bilateral and has a longer latency and duration. The present research was concerned only with observation of the R2 component and its acoustic reflex equivalent. EMG responses to the acoustic stimulus were slightly larger than those to the SO stimulus, but the two were typically close in size. The figure also shows a shorter latency to the $\mathrm{AC}$ stimulus than to the SO stimulus, a typical finding (Powers et al., 1997).
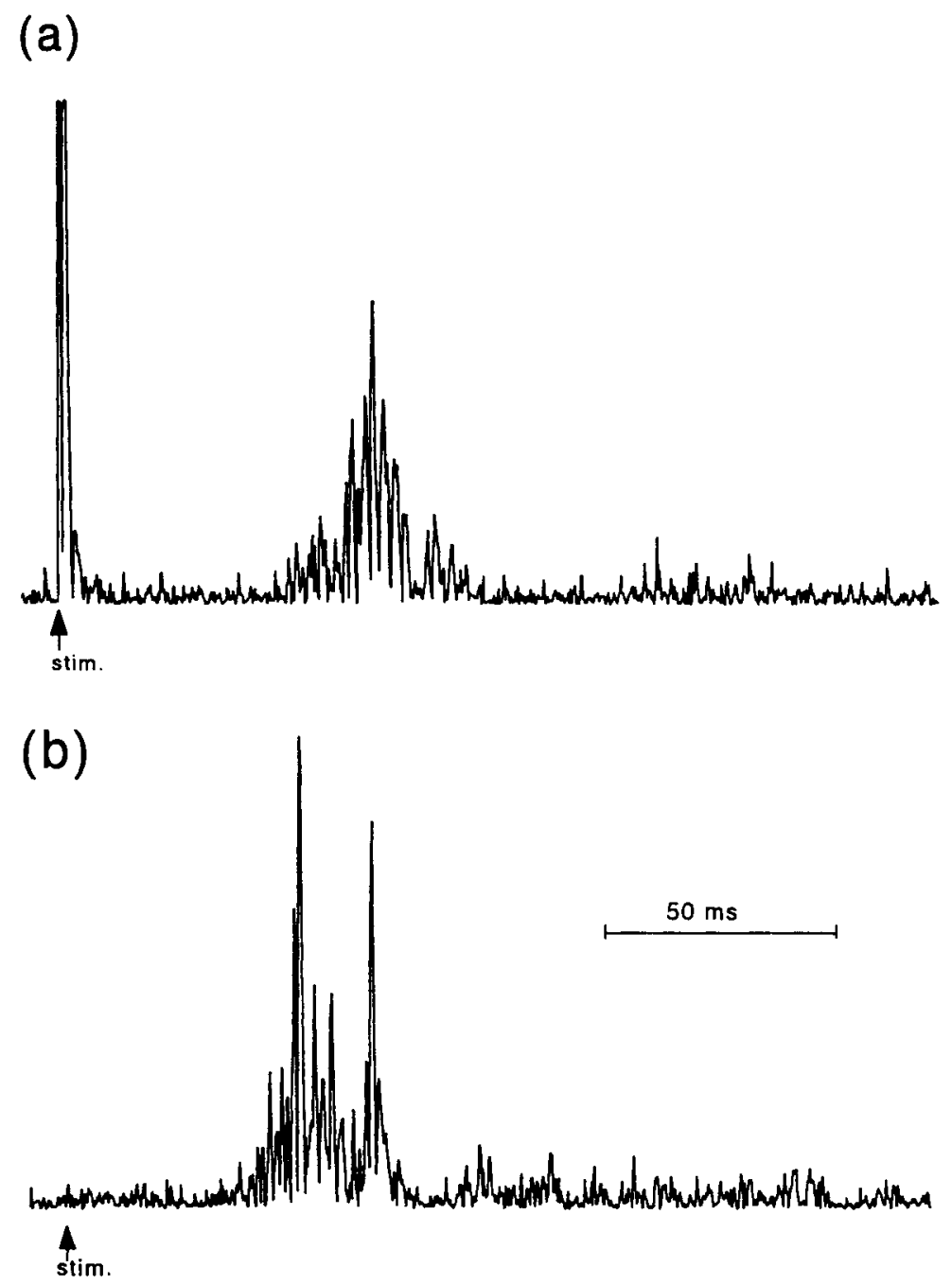

Figure 1. Example of typical EMG response for a single trial of (a) the cutaneous stimulus and (b) the acoustic stimulus, presented in a 200 -msec time window. The beginning of the stimulus presentation occurred at $10 \mathrm{msec}$ for both stimuli. 
Because there was extreme variability in the amplitude of EMG responses between subjects, the responses for each subject were transformed by dividing each trial by the first trial in the series. The data were not found to be excessively skewed, so no further transformations were performed. To reduce variability further, the 25 trials of habituation data for each subject were combined into five 5-trial blocks for statistical analysis.

Preliminary analyses. Preliminary analyses were performed to determine whether the results were affected by initial stimulus type in the ALT group. In a comparison of acoustic responses, no significant differences in habituation were found between the ALT subjects who received a cutaneous stimulus on the first trial and those who received an acoustic stimulus on the first trial $[F(1,8)=$ $2.15, p=.18 ; r_{m}=.46$, power $=.37$ ]. (All power and effect size estimations were obtained using PowPal; Gorman, Primavera, \& Allison, 1995.) Similarly, no significant differences were found for habituation to cutaneous stimuli based on stimulus of first trial $[F(1,8)=0.43$, $p=.53 ; r_{m}=.226$, power $\left.=.15\right]$. Since no differences were found between the counterbalanced subgroups of the ALT group, the data were collapsed into one group for all subsequent analyses.

SO-ONLY Versus ALT. Figure 2a shows the results for the responses to cutaneous stimuli for the SO-ONLY and ALT groups, presented as the mean proportion of first-trial response per trial. As predicted, the ALT group showed more habituation (i.e., reached a lower asymptote) than did the SO-ONLY group. In addition, the ALT group showed more rapid habituation over the first several trials.

In order to compare the rate and amount of habituation between the SO-ONLY and ALT groups for responses to cutaneous stimuli, a two-factor split-plots analysis of variance (ANOVA) with repeated measures was utilized. The two factors were group (ALT and SO-ONLY) and the repeated measures factor of trial block (1-5). The data on which this analysis was based is presented in Figure $2 b$. In this analysis and subsequent ones based on the fivetrial data, an overall group effect indicates a difference in rate of habituation between groups because the first-trial amplitude for both groups was set at 1. Both the ALT and SO-ONLY groups showed a significant amount of response decrement over trials [Pillais-Bartlett Approximation; $F(4,15)=11.95, p<.001 ; r_{m}=.872$ ], indicating that habituation occurred. The interpolation of alternated acoustic stimuli influenced habituation to cutaneous stimuli; that is, the ALT group habituated more rapidly and to a greater degree to the cutaneous stimuli than did the SO-ONLY group, as evidenced by a significant group effect $\left[F(1,18)=6.75, p=.018 ; r_{m}=.522\right]$. The group $\times$ block interaction was not significant [Pillais-Bartlett Approximation; $F(4,15)=0.116, p=.975 ; r_{m}=.173$, power $=.161]$. In Figure $2 \mathrm{a}$, one can see that the habituation rates for the two groups differ from those in the first several trials. The SO-ONLY group's mean amplitude of response actually increases over the first three

\section{(a)}

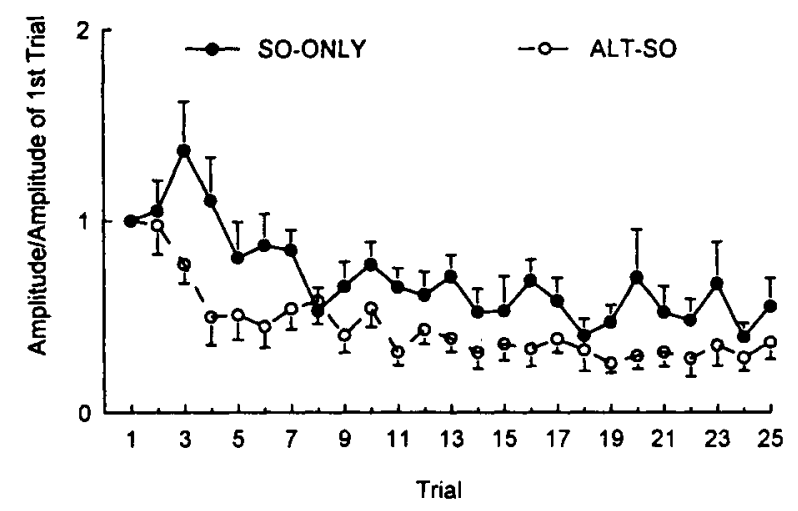

(b)

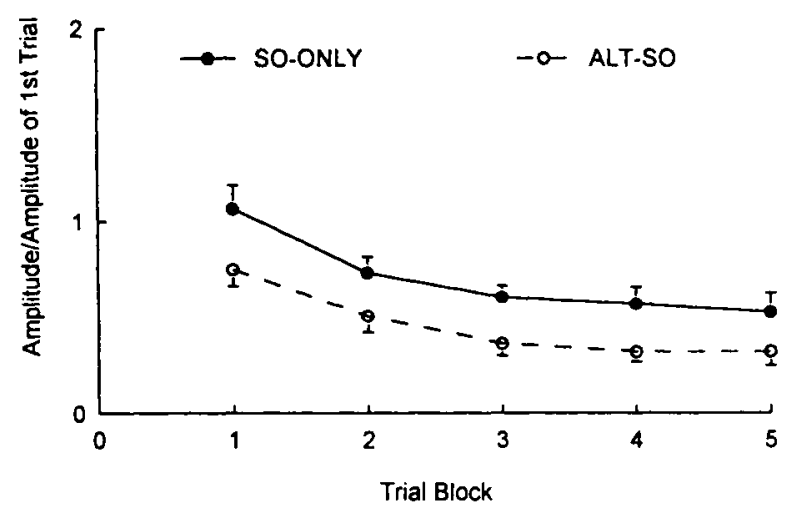

Figure 2. The responses on 25 habituation trials to cutaneous stimuli for the SO-ONLY and ALT groups. The data are presented as the mean across subjects of response amplitude divided by amplitude on the first trial, with baseline subtracted. (a) Individual trials; (b) 5-trial blocks. Error bars represent standard errors.

trials, suggesting that sensitization has occurred. In contrast, the ALT group does not display sensitization, but instead shows a rapid decrease in mean response amplitude over the first four trials, where it reaches an asymptote and decreases minimally thereafter. A comparison of the two groups over Trials 2 and 3 indicates that the response amplitudes went in opposite directions, as evidenced by a significant interaction $[F(1,18)=5.16, p=$ $\left..036 ; r_{m}=.472\right]$

AC-ONLY Versus ALT. The data for the responses to AC stimuli for the AC-ONLY and ALT groups, presented as the mean proportion of first-trial response per trial, can be seen in Figure 3a. The mean response amplitude for the AC-ONLY group appears to have decreased more rapidly than that for the ALT group. This is opposite to the trend seen in the previous SO response comparison. The differences were not significant, however. By the end of the 25 trials, the ALT and AC-ONLY groups had habituated to the same level. 
(a)

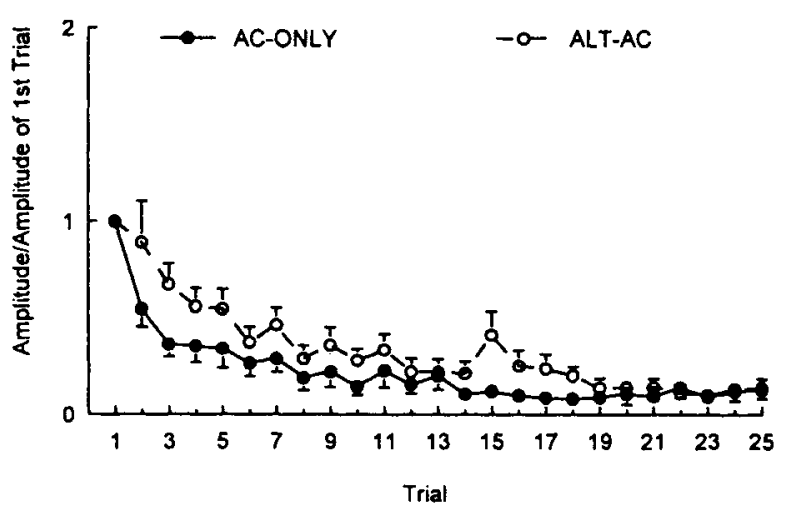

(b)

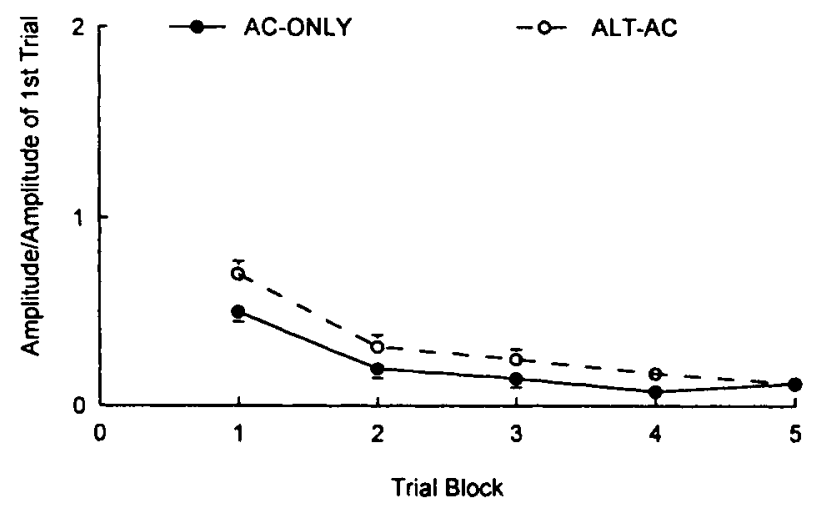

Figure 3. Responses on 25 habituation trials to acoustic stimuli for the AC-ONLY and ALT groups. The data are presented as the mean across subjects of response amplitude divided by amplitude on the first trial, with baseline subtracted. (a) Individual trials; (b) 5-trial blocks. Error bars represent standard errors.

A similar two-factor, split-plots ANOVA was used to compare the habituation rates of these two groups. Figure $3 \mathrm{~b}$ shows the data on which this analysis was based. There was a significant main effect for block [PillaisBartlett Approximation; $F(4,15)=20.65, p<.001 ; r_{m}=$ $.92]$, indicating that both groups experienced a significant decrement in mean response amplitude over trial blocks (see Figure 3a). Neither the main effect for group nor the group $\times$ block interaction was significant [for Group $\left[F(1,18)=2.14, p=.161 ; r_{m}=.326\right.$, power $=.401 ;$ for the interaction, Pillais-Bartlett Approximation; $F(4,15)=$ $1.84, p=.174 ; r_{m}=.573$, power $\left.=.787\right]$.

AC-ONLY Versus SO-ONLY. Figure 4 a shows the mean proportion of first-trial response amplitude per trial for the habituation trials of the AC-ONLY and SOONLY groups; responses to the uninterrupted series of cutaneous stimuli and responses to the uninterrupted series of acoustic stimuli are compared. The AC-ONLY group clearly habituated more quickly than the SO-ONLY group, as can be seen in Figure 4a. The SO-ONLY group showed sensitization of response, whereas the AC-ONLY group's response amplitudes diminished rapidly within a few trials. The AC-ONLY group also habituated to a greater degree than the SO-ONLY group; over 25 trials, the SO-ONLY group did not reach the same level of response decrement as the AC-ONLY group.

The comparison of habituation between the two control groups was made using a two-factor (group $\times$ block), split-plots ANOVA with repeated measures. Figure $4 b$ shows the data on which the analysis was based. Again, there was a significant main effect for block [PillaisBartlett Approximation; $F(4,15)=15.66, p<.001 ; r_{m}=$ .898 ], suggesting that both control groups habituated significantly to their respective stimuli. The main effect for group was also significant $[F(1,18)=35.07, p<.001$; $\left.r_{m}=.813\right]$, whereas the group $\times$ block interaction was not significant [Pillais-Bartlett Approximation; $F(4,15)=$ $0.266, p=.895 ; r_{m}=.257$, power $\left.=.255\right]$. Independent

(a)

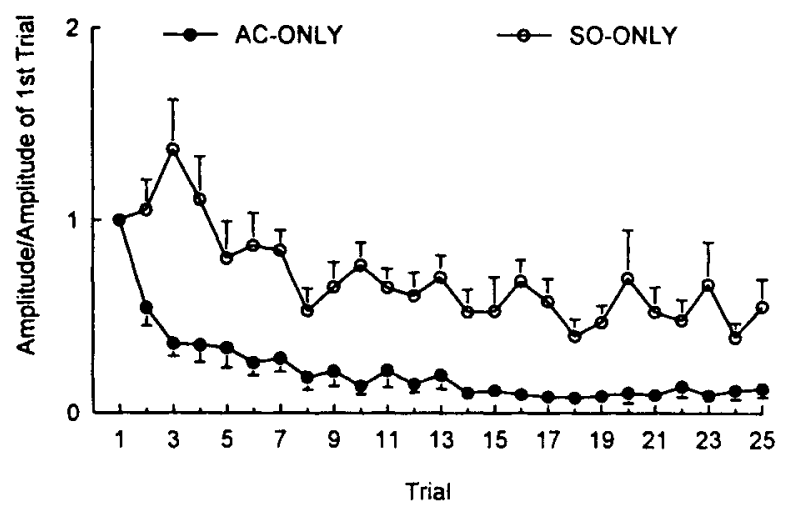

(b)

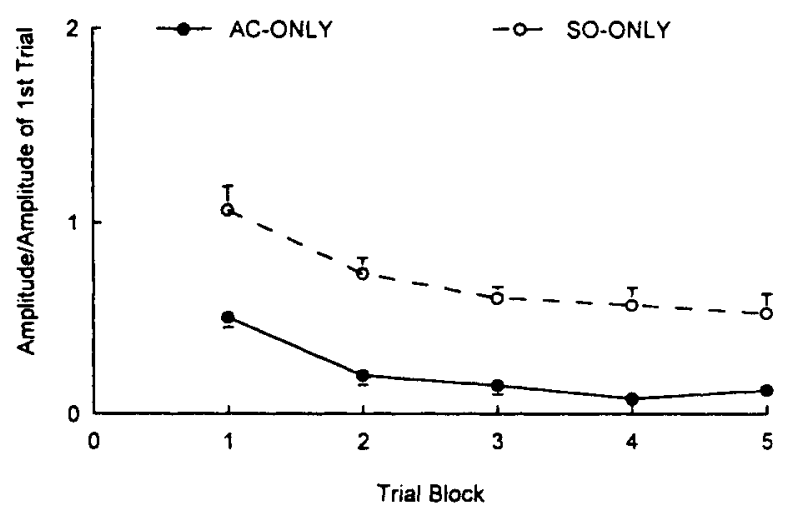

Figure 4. The responses on 25 habituation trials to acoustic and cutaneous stimuli for the AC-ONLY and SO-ONLY groups, respectively. The data are presented as the mean across subjects of response amplitude divided by amplitude on the first trial, with baseline subtracted. (a) Individual trials; (b) 5 trial blocks. Error bars represent standard errors. 
$t$ tests showed significant group differences for Block 1 $[t(18)=-3.89, p=.001]$ and for Block $5[t(18)=-3.61$, $p=.002]$, suggesting that the AC-ONLY group habituated faster initially, and to a greater degree at the end of 25 trials, than did the SO-ONLY group.

AC Versus SO within ALT group. The final analysis compared the ALT group's rate and extent of habituation to the alternating acoustic and cutaneous stimuli. As shown in Figure 5a, the trial-by-trial amplitudes of responses to cutaneous and acoustic stimuli were very similar for the same subjects. There were no differences in the rate or extent of habituation to the two stimuli. Thus, an interrupted series of cutaneous stimuli evoked habituation comparable to that evoked by an interrupted series of acoustic stimuli.

A two-factor within-subjects ANOVA was used to analyze the data presented in Figure $5 \mathrm{~b}$. The main effect for block was significant [Pillais-Bartlett Approximation; $\left.F(4,6)=19.17, p=.001 ; r_{m}=.963\right]$, indicating that the subjects in the ALT group experienced significant response decrement across trials for both stimuli (i.e., ha-

(a)

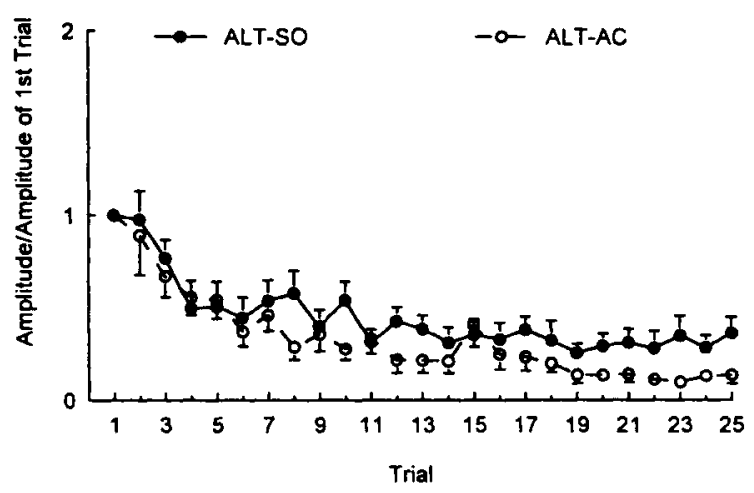

(b)

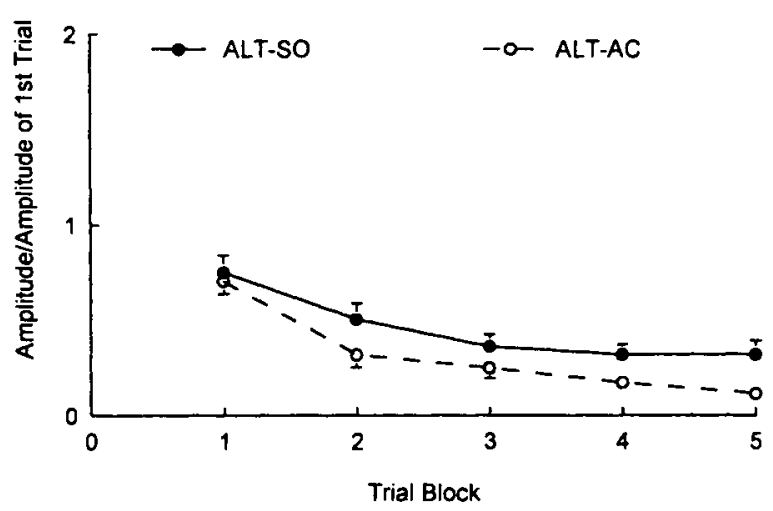

Figure 5. The responses on 25 habituation trials to acoustic and cutaneous stimuli for the subjects in the ALT group. The data are presented as the mean across subjects of response amplitude divided by amplitude on the first trial, with baseline subtracted. (a) Individual trials; (b) 5 trial blocks. Error bars represent standard errors. bituation had occurred). Neither the main effect for stimulus type $\left[F(1,9)=3.19, p=.108 ; r_{m}=.512\right.$, power $=$ .481 ] nor the stimulus $\times$ block interaction [Pillais-Bartlett Approximation; $F(4,6)=0.792, p=.571 ; r_{m}=.588$, power $=.446]$ was significant.

\section{DISCUSSION}

\section{Alternation Effects on Cutaneous Stimulation}

The present results support our primary hypothesis. The comparison between the responses of the SO-ONLY group and the cutaneous responses of the ALT group shows a main effect for group, indicating that the ALT group habituated more rapidly to the cutaneous stimuli over 25 trials than did the SO-ONLY group (see Figure 2). This finding offers support for the hypothesis that eyeblink responses to acoustic stimuli themselves stimulate the sensory receptors of the SO branch of the trigeminal nerve, the same nerve that was directly stimulated by the cutaneous (electrical) stimulus used in this and previous studies. The indirect stimulation is due to sensory feedback from lid closure. Although the closing of the eyelid does not itself elicit a blink response (because of inhibition generated by the first blink; Pellegrini, Horn, \& Evinger, 1995; Powers et al., 1997), the SO nerve has been stimulated, adding to the habituation process. Following the logic of this hypothesis, when the cutaneous and acoustic stimuli were alternately presented the subjects received twice as many stimuli to the SO nerve than the subjects in the SO-ONLY control group, in the same time span. Previous studies have consistently found that habituation rates to repeated stimuli vary inversely with the length of the ISI (Groves \& Thompson, 1970; Sanes \& Ison, 1983). In the present study, the ALT group, in essence, received SO stimulation at a $2.5-\mathrm{sec}$ ISI, whereas the SO-ONLY group received SO stimulation at a $5-\mathrm{sec}$ ISI; thus, we expected that the ALT group would habituate more rapidly than the SO-ONLY group, which it did.

\section{Alternation Effects on Acoustic Stimulation}

The results show that the ALT group did not exhibit faster habituation rates to acoustic stimuli than did the AC-ONLY group. If the ALT group had habituated more quickly, the cutaneous stimulation would have had to affect the auditory reflex arc at some point after the separate sensory pathways converged. An SO stimulus cannot directly or indirectly stimulate the auditory sensory system. The results show, however, that the ALT group did not habituate more rapidly or to a greater degree than the AC-ONLY group; therefore, the interpolated cutaneous stimuli do not enhance or accelerate habituation to the acoustic stimuli. These findings are consistent with those of two previous studies that found no cross habituation when animals or humans were first habituated to one stimulus and then were shifted to a stimulus in another modality (Dycus \& Powers, 1997; File \& Russell, 1972). As described earlier, Dycus and Powers showed that when stimuli activate only one modality, habituation 
to another modality is unaffected. File and Russell investigated cross-modal habituation of the orienting reflex in rats, using cessation of licking as a measure of the orienting response, and found no cross habituation between visual and acoustic stimuli.

In contrast, the present results are not consistent with the findings of Rimpel et al. (1982), who found transfer of habituation from one sensory modality to another. Rimpel et al. used visual (flashes of light), acoustic (tone), and cutaneous (trigeminal) stimuli to investigate habituation of the eyeblink. They found that transfer of habituation had occurred, given that response to the second scimulus type had habituated significantly faster when it had already habituated to another stimulus modality. These experimenters studied only four out of the six possible stimulus pairings: They did not use the cutaneous stimulus as a preceding stimulus for the acoustic stimulus.

Although no significant differences in the rate or degree of habituation to acoustic stimuli between the two groups were found in our experiment, Whitlow (1975), studying habituation of reflexive vasoconstriction in rabbits, and Valls-Solé, Hallett, and Alvarez (1996), studying habituation of the blink reflex to acoustic and cutaneous stimuli, found that interpolating stimuli from one modality into a series of stimuli in a different modality caused dishabituation. The many procedural differences between those studies and the present one probably account for the failure to find dishabituation effects here.

\section{Stimulus Modality Effects}

The results show that an unalternated series of acoustic stimuli evoked habituation more rapidly and to a greater degree than an unalternated series of cutaneous stimuli. As is shown in Figure 4, the AC-ONLY group habituated faster than the SO-ONLY group, as is evidenced by a significant group difference in the first trial block. The AC-ONLY group also habituated to a greater degree over 25 trials, given that there was still a significant group difference in the last trial block.

These findings are consistent with the findings of Bolino et al. (1993), who also compared habituation rates to acoustic (tones) and electrocutaneous stimulation. Bolino et al. found that subjects habituated to a greater degree to the acoustic stimulus than to the cutaneous stimulus. Although they reported that the habituation rates to the two stimuli were not significantly different, they did not adjust for the significant difference in the initial response amplitude. Their graphed data showed that the initial response amplitude to the electrocutaneous stimuli was more than twice that of the initial amplitude to the acoustic stimulus. Moreover, they employed a withinsubjects design, in which order effects could have influenced the habituation data.

\section{Effect of Alternation on Pattern of Responding}

The SO-ONLY group showed sensitization over the first three trials (see Figure 2a). This is consistent with the findings of previous studies (e.g., Dycus \& Powers, 1997; Sanes \& Ison, 1983) that have used SO stimulation as a habituating stimulus. In contrast, the SO trial series for the ALT group did not show evidence of sensitization. It seems that one of the effects of interpolating another type of stimulus within a trial series of SO stimuli is that the expected phenomenon of sensitization does not occur.

It should be noted that, as the alternation of stimuli enhanced the habituation to cutaneous stimuli in the ALT group, the disparity in habituation rates seen between the control groups was nullified; there was no significant difference in habituation for cutaneous and acoustic stimuli in the ALT group (see Figure 5a).

\section{Conclusion}

The present results are consistent with a large body of data that has shown that habituation takes place on the sensory side of a reflex (e.g., Davis, Parisi, Gendelman, Tischler, \& Kehne, 1982; Dycus \& Powers, 1997). Thus, in the alternating group, blinks to acoustic stimuli are not affected by cutaneous stimulation or by the fact that twice as many responses are elicited. However, an effect of presentation of acoustic stimuli is seen in the cutaneous modality, in which a habituating acoustic stimulus leads to eyeblinks that produce cutaneous stimulation.

\section{REFERENCES}

Bolino, F., Di Michele, V., Manna, V., Di Cicco, L., Isidori, M. V., \& CassaCchia, M. (1993). Acoustic and electrically elicited startle reaction: Similar patterns of habituation and reflex modifications in humans. International Journal of Neuroscience, 73, 13-21.

Davis, M., Parisi. T., Gendelman, D. S.. Tischler, M.. \& Kehne. J. H. (1982). Habituation and sensitization of startle reflex elicited electrically from the brainstem. Science, 218, 688-690.

Drcus. W. A., \& Powers. A. S. (1997). Eyeblink cross-habituation between tactile and acoustic systems in humans. Psychobiology, 25, 66-70.

Evinger, C., Shaw, M. D., Peck, C. K., Manning, K. A.. \& Baker, R. (1984). Blinking and associated eye movements in humans, guinea pigs, and rabbits. Journal of Neurophysiology, 52, 323-339.

File. S. E., \& Russell. I. S. (1972). Specificity and savings of behavioural habituation over a series of intra- and inter-modal stimuli. Quarterly Journal of Experimental Psychology. 24, 465-473.

Gorman. B. S., Primavera, L. H., \& Allison, D. B. (1995). POWPal: A program for estimating effect sizes, statistical power, and sample sizes. Educational \& Psychological Measurement, 55, 773-776.

Graham. F. K. (1975). The more oi less startling effects of weak pre stimulation. Psychophysiology, 12, 238-248

Groves, P. M., \& Thompson, R. F. (1970). Habituation: A dual process theory. Psychological Review, 77, 419-450.

KANDEL, E. R. (1978). A cell-hiological approach to learning. Bethesda. MD: Grass Lecture Monographs.

KugelberG, E. (1952). Facial reflexes. Brain, 75, 385-396.

Pellegrini, J. J., Horn, A. K. E., \& Evinger, C. (1995). The trigeminally evoked blink reflex: I. Neuronal circuits. Experimental Brain Research, 107, 166-180.

Powers, A. S.. Schic atano, E. J., Basso, M. A. \& Evinger, C. (1997) To blink or not to blink: Inhibition and facilitation of reflex blinks Experimental Brain Research, 113, 283-290.

ReITER, L. A., \& ISON, J. R. (1977). Inhibition of the human eyeblink reflex: An evaluation of the sensitivity of the Wendt-Yerkes method for threshold detection. Journal of Experimental Psychology: Human Perception \& Performance, 3, 325-336.

Rimpel, J.. Geyer, D.. \& Hopf, H. C. (1982). Changes in the blink re- 
sponses to combined trigeminal, acoustic, and visual repetitive stimulation, studied in the human subject. Electroencephalography \& Clinical Neurophysiology, 54, 552-560.

SANES, J. N., \& ISON, J. R. (1983). Habituation and sensitization of components of the human eyeblink reflex. Behavioral Neuroscience, 97 , 833-836.

Sokolov, E. N. (1960). Neuronal models and the orienting reflex. In M. A. B. Brazier (Ed.), The central nervous system and behavior (pp. 187-286). New York: Josiah Macy Foundation.

TACKMAnN, W., EtTlin, T., \& BARTH, R. (1982). Blink reflexes elicited by electrical, acoustic, and visual stimuli: I. Normal values and possible anatomical pathways. European Neurology, 21, 210-216.
Valls-Solé, J., Hallett, M., \& Alvarez, R. (1996). Postpulse effects on blink reflex responses. Electroencephalography \& Clinical Neurophysiology, 101, 504-510.

WAGNER, A. R. (1979). Habituation and memory. In A. Dickinson \& R. A. Boakes (Eds.), Mechanisms of learning and motivation (pp. 5382). Hillsdale, NJ: Erlbaum.

WhitLOW, J. W., JR. (1975). Short-term memory in habituation and dishabituation. Journal of Experimental Psychology: Animal Behavior Processes, 1, 189-206.

(Manuscript received March 21, 2000; revision accepted for publication October 18,2000 .) 